\title{
Identification of neon in FUSE and VLT spectra of extremely hot hydrogen-deficient (pre-) white dwarfs ${ }^{\star} \star \star$
}

\author{
K. Werner ${ }^{1}$, T. Rauch ${ }^{1,2}$, E. Reiff ${ }^{1}$, J. W. Kruk ${ }^{3}$, and R. Napiwotzki ${ }^{4}$ \\ ${ }^{1}$ Institut für Astronomie und Astrophysik, Universität Tübingen, Sand 1, 72076 Tübingen, Germany \\ e-mail: werner@astro.uni-tuebingen.de \\ ${ }^{2}$ Dr.-Remeis-Sternwarte, Universität Erlangen-Nürnberg, Sternwartstraße 7, 96049 Bamberg, Germany \\ 3 Department of Physics and Astronomy, Johns Hopkins University, Baltimore, MD 21218, USA \\ 4 Department of Physics and Astronomy, University of Leicester, University Road, Leicester, LE1 7RH, UK
}

Received 26 April 2004 / Accepted 16 July 2004

\begin{abstract}
One of the strongest absorption lines observed in far-ultraviolet FUSE spectra of many PG 1159 stars remained unidentified up to now. We show that this line, located at $973.3 \AA$, stems from Ne VII. We also present new optical highresolution spectra of PG 1159 stars, obtained with the ESO VLT, which cover the Ne VII $3644 \AA$ A line and a newly identified Ne VII multiplet in the 3850-3910 Å region. We compare the observed neon lines with NLTE models and conclude a substantial neon overabundance in a number of objects. Although a detailed analysis is still to be performed in order to compare quantitatively the abundances with evolutionary theory predictions, this corroborates the idea that the PG 1159 stars and their immediate progenitors, the [WC]-type nuclei of planetary nebulae, display intershell matter of their precursor AGB stars. Possibly as the consequence of a late He-shell flash, H-deficient and (s-processed) Fe-depleted matter, that is strongly enriched by $3 \alpha$-processed elements $(\mathrm{C}, \mathrm{O}, \mathrm{Ne})$, is dredged up to the surface. Hence, a detailed study of the element abundance patterns in these peculiar stars gives the unique possibility to probe mixing and nucleosynthesis processes in the precursor AGB stars.
\end{abstract}

Key words. stars: abundances - stars: atmospheres - stars: evolution - stars: AGB and post-AGB - stars: white dwarfs

\section{Introduction}

The PG 1159 stars are hot hydrogen-deficient (pre-) white dwarfs $\left(T_{\text {eff }}=75000-200000 \mathrm{~K}, \log g=5.5-8(\mathrm{cgs})\right.$; Werner 2001). They are probably the outcome of a late helium-shell flash, a phenomenon that drives the currently observed fast evolutionary rates of three well-known objects (FG Sge, Sakurai's object, V605 Aql). Flash-induced envelope mixing produces a H-deficient stellar surface. The photospheric composition then essentially reflects that of the region between the $\mathrm{H}$-and He-burning shells in the precursor AGB star. The He-shell flash transforms the star back to an AGB star and the subsequent, second post-AGB evolution explains the existence of Wolf-Rayet central stars of planetary nebulae and their successors, the PG 1159 stars.

The detection of a Ne VII line at $3644 \AA$ in three PG 1159 stars (RX J2117.1+3412, NGC 246, K1-16; Werner \& Rauch 1994, WR94) and the derived, high abundance of $\mathrm{Ne}=2 \%$ (by mass; i.e. 20 times solar) strengthened the view that these

* Based on observations made with the NASA-CNES-CSA Far Ultraviolet Spectroscopic Explorer. FUSE is operated for NASA by the Johns Hopkins University under NASA contract NAS5-32985.

$\star \star$ Based on observations at the Paranal Observatory of the European Southern Observatory for programs Nos. 165.H-0588(A), 167.D-0407(A), and 69.D-0719(A). stars display intershell matter strongly enriched by $\mathrm{Ne}$ from $3 \alpha$ burning. The next positive result of a search for neon was obtained from EUVE spectra of H1504+65, which showed that very strong and broad Ne VII and O VI lines dominate the soft X-ray region (100-130 $\AA$; Werner \& Wolff 1999). From these data and the Ne VII $3644 \AA$ line in a Keck spectrum, a neon abundance of 2-5\% was derived. Dozens of lines from Ne VI-Ne VIII were later detected in the Chandra spectrum of H1504+65, confirming this result (Werner et al. 2004, W04). This recent analysis also took advantage of FUSE (Far Ultraviolet Spectroscopic Explorer) spectra, in which we detected for the very first time in any stellar spectrum another Ne VII line, located at $973 \AA$ A. It is among the strongest absorption features in that FUSE spectrum.

In this paper we present the results of our systematic search for this newly identified FUV line in FUSE spectra of a number of PG 1159 stars. We also present new high-resolution optical spectra obtained with ESO's VLT (Very Large Telescope), which allow for a much deeper search for the Ne VII $3644 \AA$ line, compared to our earlier WR94 investigation. We also announce the first detection of a Ne VII multiplet at 3850-3910 ̊. It will be shown that the Ne VII $973 \AA$ line is much stronger than the optical neon lines, and that it can still be observed in objects with photospheric parameters for which the optical lines are no 
Table 1. Atmospheric parameters of the program stars as taken from the literature. The neon abundance has been set to $2 \%$. Abundances are given in $\%$ mass fraction.

\begin{tabular}{lrrrrrrr}
\hline \hline Object & $\begin{array}{r}T_{\text {eff }} \\
{[\mathrm{kK}]}\end{array}$ & $\begin{array}{l}\log g \\
(\mathrm{cgs})\end{array}$ & $\mathrm{H}$ & $\mathrm{He}$ & $\mathrm{C}$ & $\mathrm{O}$ & Ref. \\
\hline RX J0122.9-7521 & 180 & 7.5 & & 66 & 21 & 11 & $\mathrm{~A}$ \\
RX J2117.1+3412 & 170 & 6.0 & & 38 & 54 & 6 & $\mathrm{~B}$ \\
PG 1520+525 & 150 & 7.5 & & 43 & 38 & 17 & $\mathrm{~J}$ \\
PG 1144+005 & 150 & 6.5 & & 38 & 58 & 2 & $\mathrm{C}$ \\
NGC 246 & 150 & 5.7 & & 62 & 30 & 6 & $\mathrm{~F}$ \\
PG 1159-035 & 140 & 7.0 & & 33 & 48 & 17 & $\mathrm{I}, \mathrm{J}$ \\
Abell 21 & 140 & 6.5 & & 33 & 48 & 17 & $\mathrm{~F}$ \\
K1-16 & 140 & 6.4 & & 33 & 48 & 17 & $\mathrm{G}$ \\
Longmore 3 & 140 & 6.3 & & 38 & 54 & 6 & $\mathrm{~F}$ \\
PG 1151-029 & 140 & 6.0 & & 33 & 48 & 17 & $\mathrm{~F}$ \\
Longmore 4 & 120 & 5.5 & & 45 & 42 & 11 & $\mathrm{D}$ \\
PG 1424+535 & 110 & 7.0 & & 49 & 43 & 6 & $\mathrm{~J}$ \\
Abell 43 & 110 & 5.7 & 35 & 41 & 21 & 1 & $\mathrm{E}$ \\
NGC 7094 & 110 & 5.7 & 35 & 41 & 21 & 1 & $\mathrm{E}$ \\
Abell 30 & 110 & 5.5 & & 33 & 48 & 17 & $\mathrm{H}$ \\
MCT0130-1937 & 90 & 7.5 & & 73 & 22 & 3 & $\mathrm{~J}$ \\
PG 1707+427 & 85 & 7.5 & & 43 & 38 & 17 & $\mathrm{~J}$ \\
\hline
\end{tabular}

References in last column: A: Werner et al. (1996a); B: Werner et al. (1996b); C: Werner \& Heber (1991); D: Werner et al. (1992); E: Dreizler et al. (1997); F: Rauch \& Werner (1997); G: Kruk \& Werner (1998); H: Werner et al. (2003a); I: Werner et al. (1991); J: Dreizler \& Heber (1998).

longer detectable. In contrast to the optical lines, the FUV line is even detectable at a solar abundance level and can thus be used as a unique tool for the neon abundance determination in very hot H-rich central stars.

In Sect. 2 we describe our observations, followed by a presentation of our model atmospheres in Sect.3. In Sect. 4 we compare observed and computed Ne VII line profiles. We discuss implications of our results on interior processes in the precursor AGB stars in the final Sect. 5.

\section{FUSE and VLT observations}

Our sample consists of 17 PG 1159 stars, which represent about $50 \%$ of all known objects in this spectroscopic class. Their basic atmospheric parameters are listed in Table 1. The sample contains two so-called hybrid-PG 1159 stars, which display hydrogen Balmer lines in their spectra (Abell 43 and NGC 7094). Traces of nitrogen (typically $1 \%$ by mass) were discovered in some PG 1159 stars. This is neglected in Table 1, but will be discussed in the last section.

In Table 2 we list the FUSE and VLT spectra available for our program stars. The FUSE instrument consists of four coaligned telescopes, each with a prime-focus spectrograph. Descriptions of the FUSE instrument, and channel alignment and wavelength calibration issues are given by Moos et al. (2000) and Sahnow et al. (2000). The LWRS spectrograph apertures were used for all observations other than $\mathrm{K} 1-16$, which was observed in all 3 apertures. As a result, the zero-point of the wavelength scale is uncertain to within
Table 2. Log of FUSE and VLT observations of our program stars. VLT observations were performed with two different instrumental setups (see text).

\begin{tabular}{l|cc|c|c}
\hline \hline Object & $\begin{array}{c}\text { FUSE } \\
\text { dataset } \\
\text { name }\end{array}$ & $\begin{array}{c}t_{\text {exp }} \\
{[\mathrm{min}]}\end{array}$ & $\begin{array}{c}t_{\text {exp }} \\
{[\mathrm{min}]}\end{array}$ & $\begin{array}{c}t_{\text {exp }} \\
{[\mathrm{min}]}\end{array}$ \\
\hline RX J0122.9-7521 & - & - & 10 & - \\
RX J2117.1+3412 & P1320501 & 137 & - & - \\
PG 1520+525 & P1320101 & 81 & - & - \\
PG 1144+005 & P1320201 & 114 & 10 & 60 \\
NGC 246 & - & - & 5 & - \\
PG 1159-035 & Q1090101 & 105 & 5 & 60 \\
Abell 21 & - & - & 10 & - \\
K1-16 & M1031001-8 & 665 & - & - \\
Longmore 3 & - & - & 5 & - \\
PG 1151-029 & - & - & 10 & - \\
Longmore 4 & B0230201 & 434 & 10 & 60 \\
PG 1424+535 & P1320301 & 185 & - & - \\
Abell 43 & - & - & 5 & - \\
NGC 7094 & - & - & 5 & - \\
Abell 30 & - & - & 5 & - \\
MCT0130-1937 & - & - & 10 & - \\
PG 1707+427 & P1320401 & 243 & - & - \\
\hline
\end{tabular}

about $\pm 0.15 \AA$ A. All exposures were photometric, or nearly so, in all channels for the LWRS observations. Exposures in the HIRS and MDRS aperture K1-16 observations that had less than $50 \%$ peak flux were discarded; the rest were renormalized to match the LWRS data. All data were obtained in timetag mode except for RX J2117.1+3412, which was observed in histogram mode, and all were processed with CALFUSE v2.4. Spectra from individual exposures were coaligned in wavelength on a channel by channel basis using narrow interstellar absorption lines, averaged weighted by exposure time, and then shifted to put the photospheric O VI features at zero velocity.

Figure 1 displays the FUV spectra around the newly identified Ne VII line; an expanded view is shown in Figs. 2 and 3. They have been shifted to the rest wavelengths of the photospheric lines, using the O VI resonance line doublet. We have excluded available FUSE spectra of NGC 7094 and Abell 78 from our analysis, because the region around the Ne VII line is badly contaminated by the extremely broad P Cygni-shaped profile of the $\mathrm{C}$ III resonance line as well as by a damped $\mathrm{H}_{2}$ profile. Their analysis requires detailed wind modeling which is beyond the scope of this paper. The FUSE spectrum of another object (HS 2324+397) has been excluded from our analysis because of poor $\mathrm{S} / \mathrm{N}$ and very strong contamination by interstellar $\mathrm{H}_{2}$ absorption lines.

The Ne VII $973 \AA$ line is potentially blended with two interstellar $\mathrm{H}_{2}$ lines: the Lyman band $\mathrm{P}(1)_{11-0} 973.342 \AA$ and the Werner band $\mathrm{P}(4)_{2-0} 973.452 \AA$ transitions. The strengths and relative velocities of these features were determined from fits to the numerous other $\mathrm{H}_{2}$ absorption lines in the FUSE bandpass, along with the common low-ionization atomic species 


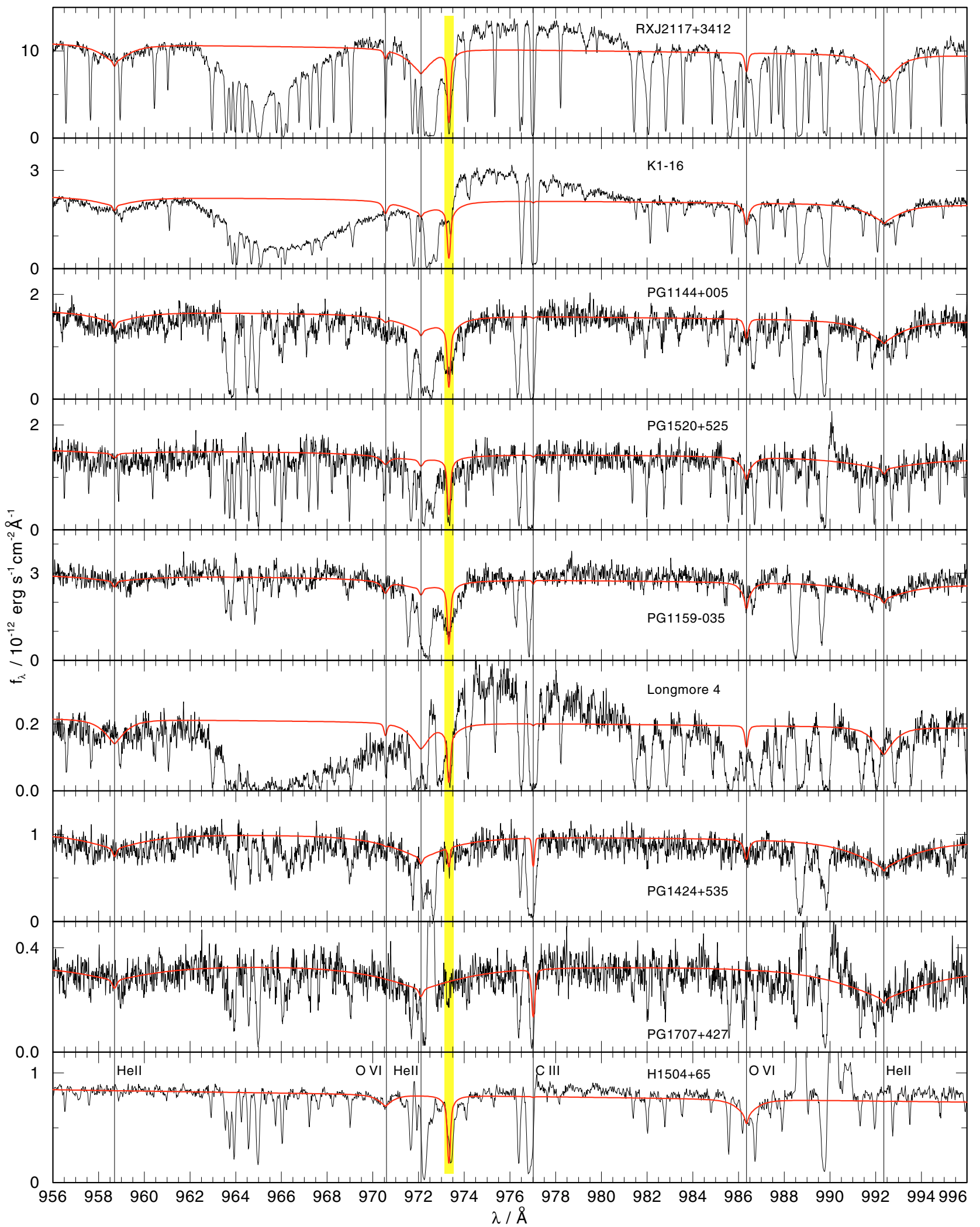

Fig. 1. Detail from FUSE spectra of PG 1159 stars showing the vicinity of the newly identified Ne VII line at $973.3 \AA$ (indicated by the vertical shaded bar). RX J2117.1+3412, K1-16, and Longmore 4 display broad and shallow P Cyg profiles from the C III $977 \AA$ resonance line. All other features, except for the labeled He II and O VI lines, are of interstellar origin. The narrow emission features are terrestrial airglow. The spectra are smoothed by Gaussians with $0.02 \AA F W H M$. Overplotted are model atmosphere spectra $(\mathrm{Ne}=2 \%)$, folded with $0.1 \AA F W H M$ Gaussians to match the instrumental resolution. 


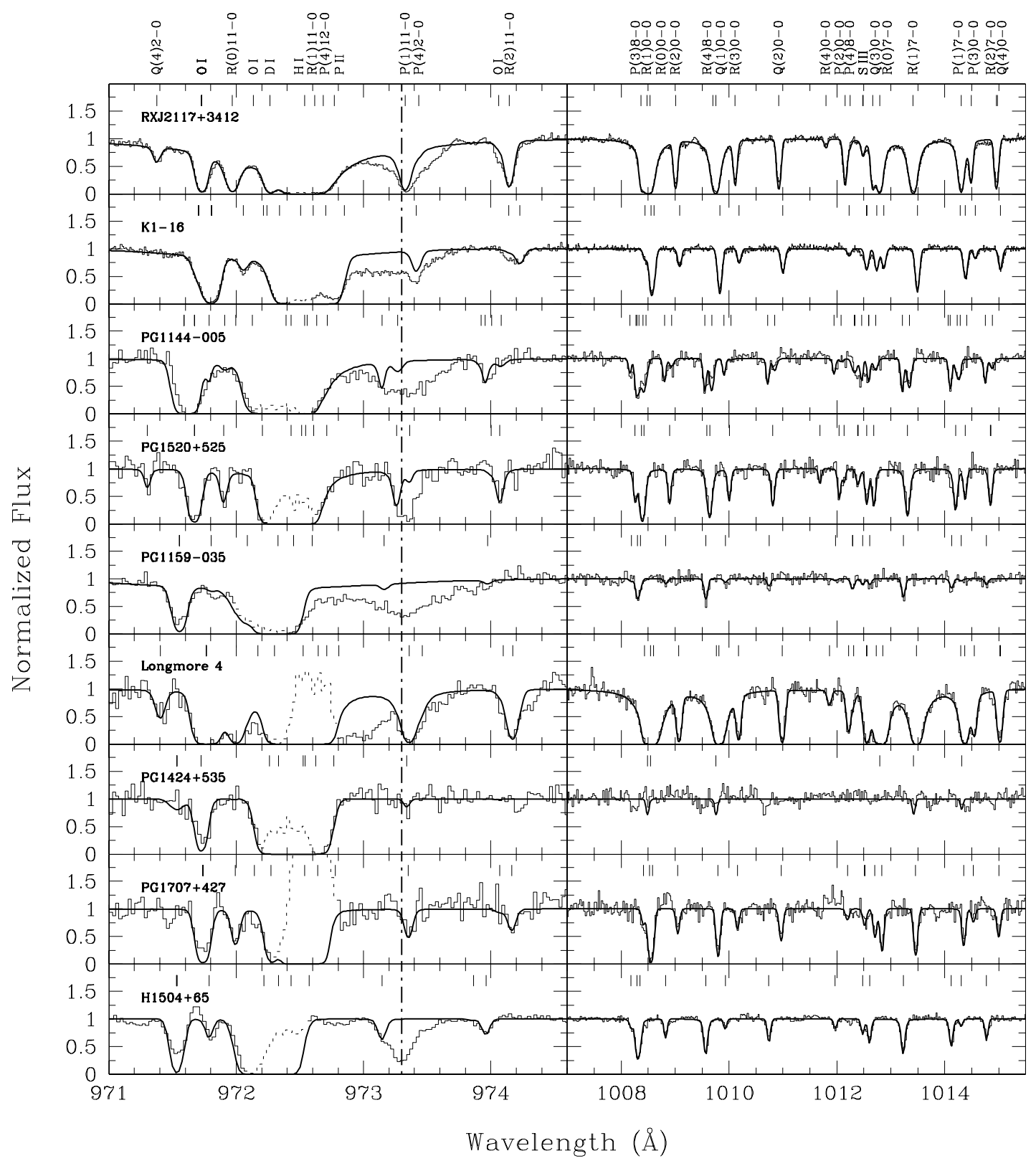

Fig. 2. Detail from FUSE spectra of PG 1159 stars showing the modeled ISM absorption. The data are plotted as the thin histogram and the ISM model is the smooth heavy line. The spectra were binned by 2 pixels in the top 2 panels and by 4 pixels $(\sim 0.027 \AA)$ in the others for plotting purposes. The panels on the left show the region in the vicinity of the newly-identified Ne VII line at $973.3 \AA$ (indicated by the vertical dot-dashed line); the panels on the right show a representative sample of $\mathrm{H}_{2}$ absorption. The ISM features are labeled at the top of the figure and marked in each plot with tickmarks. The spectra were shifted to place the photospheric O VI absorption at rest, so the ISM features appear with varying velocity offsets. Airglow Ly $\gamma$ emission is plotted with a dotted line; in some spectra O I $971.738 \AA$ airglow emission partially fills-in the corresponding IS absorption.

(O I, N I, ArI, C II, Fe II, etc.). The SiC2a, LiF1a, and LiF2a spectra were used for the fits, as they had the best signal-tonoise. Fitting was performed with the program Owens.f written by Martin Lemoine; for a discussion of the fitting procedure see Kruk et al. (2002) or Hébrard et al. (2002). Atomic data used in the fits were taken from Morton (2003), and molecular data were taken from Abgrall et al. (1993a,b). The $\mathrm{P}(4)_{2-0}$ transition is rarely significant: the column density of $\mathrm{H}_{2}$ in the $J=4$ state is typically far less than in $J=1$, and absorption by $\mathrm{H}_{2}$ from the $J=4$ level was detected only for four of the nine stars. Because of the complications associated with modeling the broad wind features in several of the stars, the stellar continuum was treated in the ISM models by fitting a fourth-order polynomial over a wide region and including broad He II profiles when appropriate. The results of the fits are shown in Fig. 2 for the vicinity of the Ne VII line, and for a region spanning a representative sample of $\mathrm{H}_{2}$ lines. This latter region includes the strongest Lyman band transitions and absorption from all $J$-levels found in the spectra. Fits of comparable quality are obtained throughout the FUSE bandpass, so the modeled $\mathrm{H}_{2}$ absorption in the vicinity 


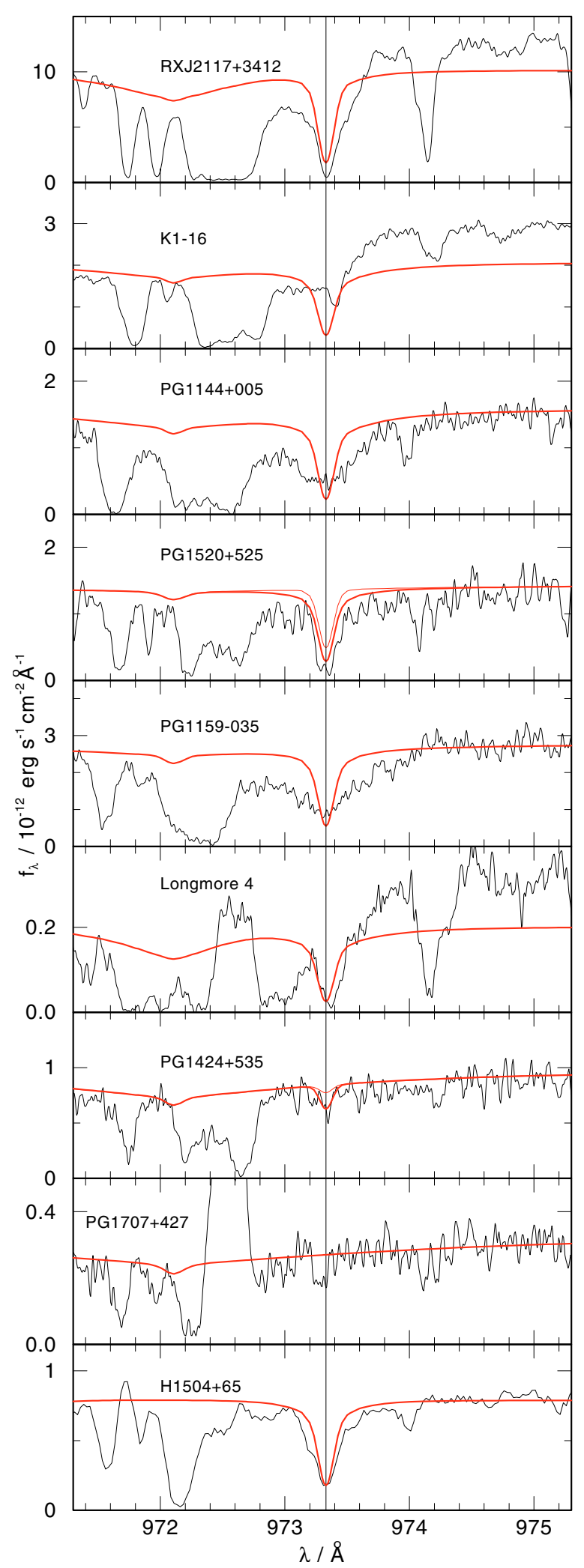

Fig. 3. Expanded view of Fig. 1, centered around the Ne VII $973.3 \AA$ line. For PG $1520+525$ and PG $1424+535$ we overplotted a second synthetic line profile (thin line), computed with a solar Ne abundance $(0.1 \%)$.

of $973.3 \AA$ should be reliable. Results for individual lines of sight are discussed in Sect. 4.1 below.
The optical spectra presented here were taken between July 2000 and August 2002 in the course of the ESO Supernovae Ia Progenitor Survey (SPY), see Table 2 (VLT/1 column). SPY is a high-resolution spectroscopic survey for radial velocity variable white dwarfs (Napiwotzki et al. 2001, 2003). Spectra were taken with the high resolution UV-Visual Echelle Spectrograph (UVES) of the UT2 telescope (Kueyen) of the ESO VLT. The SPY instrument setup (Dichroic 1, central wavelengths $3900 \AA$ and $5640 \AA$ ) used UVES in a dichroic mode and nearly complete spectral coverage from $3200 \AA$ to $6650 \AA$ with only two $\approx 80 \AA$ wide gaps at $4580 \AA$ and $5640 \AA$ is achieved. SPY was implemented as a service mode program, which took advantage of those observing conditions, which are not usable by most other programs (moon, bad seeing, clouds). A wide slit $\left(2.1^{\prime \prime}\right)$ is used to minimize slit losses and a $2 \times 2$ binning is applied to the CCDs to reduce read-out noise. Our wide slit reduces the spectral resolution to $R=18500(0.2 \AA$ at $3600 \AA)$ or better, if seeing disks were smaller than the slit width.

The spectra were reduced with a procedure developed by Karl (in preparation) using the ESO MIDAS software package, partly based on routines from the UVES pipeline reduction software provided by ESO (Ballester et al. 2003). Since the sampling of the spectra is much higher than needed for our analysis, we rebinned the spectra to $0.1 \AA$ stepsize; this considerably improved the signal-to-noise ratio with a negligible degradation of the resolution. Figures 4 and 7 display details of the spectra near the Ne VII $3644 \AA$ and 3850-3910 $\AA$ lines, respectively.

As part of another observation program, VLT/UVES spectra with significantly higher spectral resolution and integration time have been taken of PG 1159-035, PG 1144+005, and Longmore 4 between April and July 2002 (see Table 2, VLT/2 column). We used the standard DIC1 (346) setup and a slit width of $0.6^{\prime \prime}$ and arrive at a resolving power $R \approx 75000(0.05 \AA$ at $3600 \AA)$. The spectra were subject to the standard pipeline reduction provided by ESO. They cover the wavelength regions 3300-3870, , 4800-5755 , and 5835-6805 $\AA$. Figure 5 displays these spectra near the Ne VII $3644 \AA$ line.

\section{Model atmosphere calculations}

For each program star we calculated a single model atmosphere with parameters obtained from the literature (Table 1). It is beyond the scope of this paper to improve these parameters by detailed model grid calculations and line profile fits for the complete wavelength range covered by the FUSE and VLT spectra. This is deferred to an extensive analysis which has only just begun.

Line blanketed NLTE model atmospheres were computed using our PRO2 code (Werner et al. 2003b). The models are in hydrostatic and radiative equilibrium, hence, they cannot reproduce the P Cygni shaped profile of the C III resonance line observed in three objects. Detailed wind modeling will be necessary in future analyses. The atomic models account for the most abundant elements in PG 1159 stars, namely, helium, carbon, oxygen, and neon. Mass fractions were as shown in Table 1, except that the neon abundance was set to $2 \%$. We do not take 

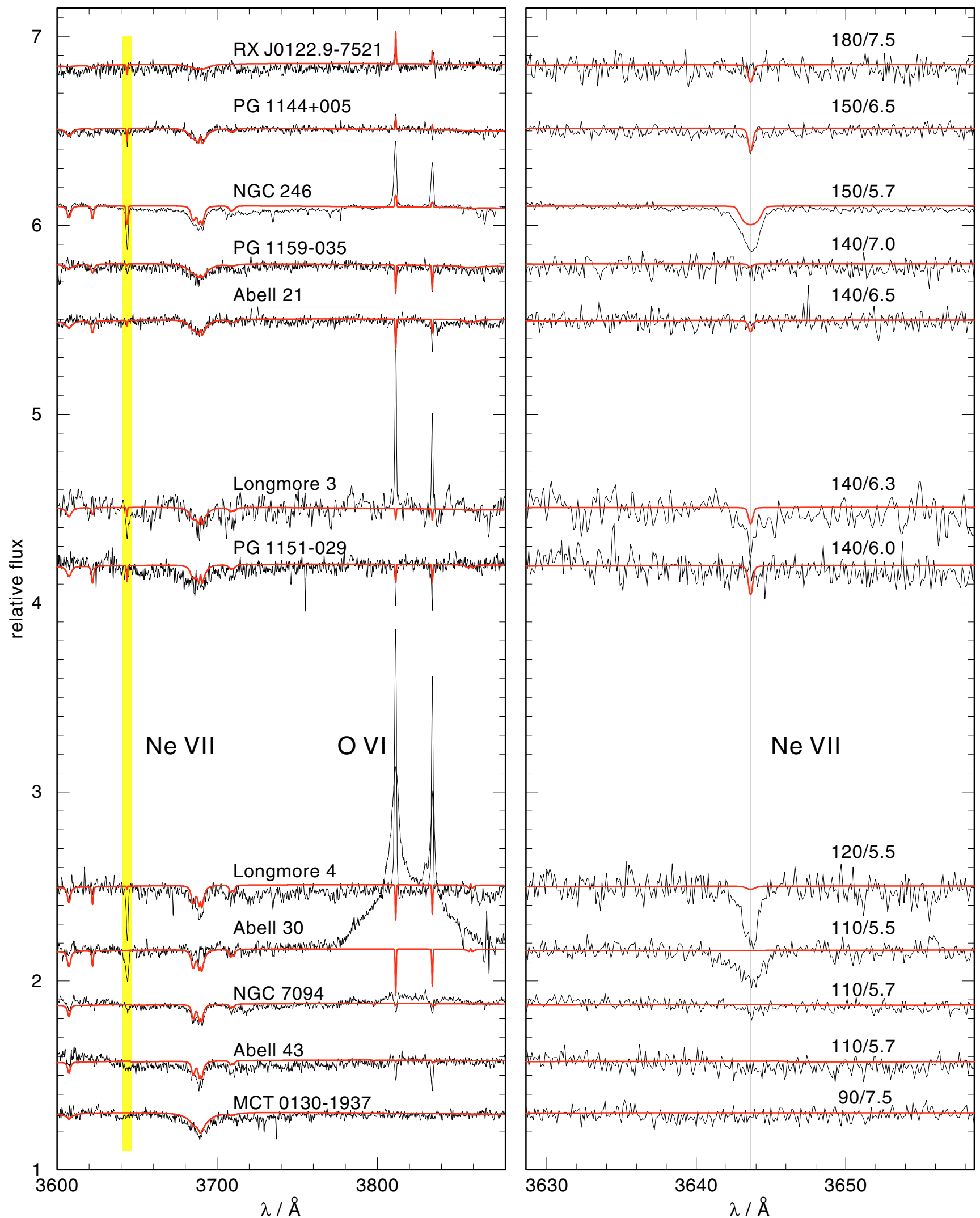

Fig. 4. Left: VLT spectra of PG 1159 stars in the vicinity of the Ne VII $3644 \AA$ line (indicated by the vertical shaded bar) and the O vI $3 \mathrm{~s}-3 \mathrm{p}$ doublet at 3811/3834 A. Right: expanded view centered around the Ne VII line. Overplotted are model spectra with $\mathrm{Ne}=2 \%$, labeled with their $T_{\text {eff }}$ (in $\mathrm{kK}$ ) and $\log g$. Smoothing of observed spectra with Gaussians (FWHM given): left panel: 0.4-0.7 $\AA$, right panel: no smoothing, except for Longmore $3(0.2 \AA)$. The model spectra are smoothed accordingly. The NGC 246 model is also rotationally broadened $\left(70 \mathrm{~km} \mathrm{~s}^{-1}\right)$. 


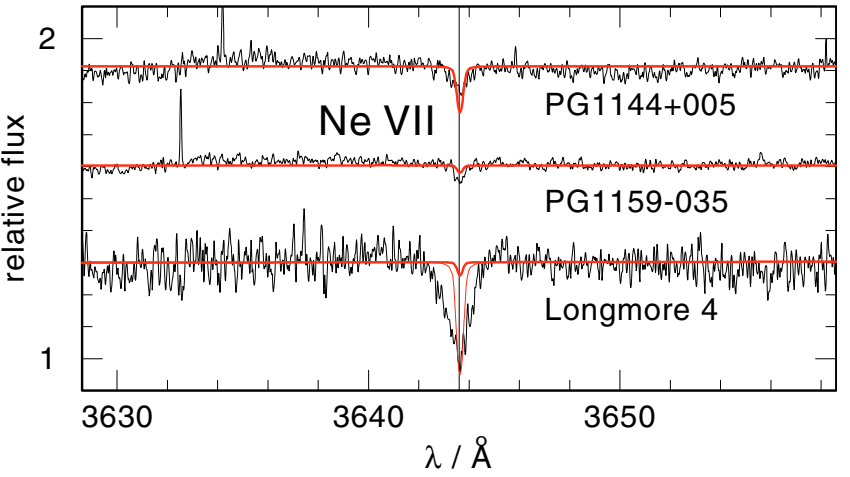

Fig. 5. Second set of VLT spectra of three PG 1159 stars in the vicinity of the Ne VII $3644 \AA$ line, taken with higher spectral resolution. In comparison with the first set of spectra from these stars (Fig. 4), we can now clearly identify the Ne VII line in PG 1159-035. Overplotted are the same model spectra as in Fig. 4. A second model is overplotted with Longmore 4, namely the NGC 246 model from Fig. 4 (but unrotated). Smoothing of observed and computed spectra is performed with $0.05 \AA$ and $0.07 \AA$ Gaussians, respectively.

into account the trace nitrogen discovered in some of our program stars. This does not affect our results obtained here. For more details on atomic models, we refer to W04.

Here we present the detection of the Ne VII $973.3 \AA$ absorption line $2 \mathrm{p}^{1} \mathrm{P}^{\mathrm{o}}-2 \mathrm{p}^{2}{ }^{1} \mathrm{D}$ in several PG 1159 stars. The search was initiated by the very first detection of this line in H1504+65 (W04). There is some uncertainty in the literature concerning the accurate wavelength position of this line. The first measurement was published by Johnston \& Kunze (1971, JK69) who give 973.6 ̊. Lang (1983) quotes $973.33 \AA$ (without reference) and this value is also found in the Chianti database (Young et al. 2003). According to Kramida (NIST, private communication) the best measurement for this line was done by Lindeberg (1972). The measured wavelength is $973.302 \pm 0.005 \AA$. For our synthetic spectra we have adopted $973.33 \AA$.

In Table 3 we list the UV/optical Ne VII lines detected in PG 1159 stars. Besides the lines discussed here, we include for completeness the Ne VII triplet around $1992 \AA$ which was identified in one object (NGC 246, WR94).

Our model calculations show that the $973 \AA$ line of Ne VII is always much stronger than the optical $3634 \AA$ line. This is because the excitation energies of the (lower) levels involved are very different (see Table 3 ). The level excitation energy of the $973 \AA$ line corresponds to an excitation temperature of $310 \mathrm{kK}$, while that of the $3634 \AA$ line corresponds to $1400 \mathrm{kK}$. As a consequence, the population density of the lower level of the FUV line is by many orders of magnitude larger than that of the optical line (at least by four orders, depending on the model parameters and the depth in the atmosphere). Therefore in some objects the FUV line can be prominent while the optical line is not detectable. It is obvious that the Ne VII $973 \AA$ line is very strong in many of our program stars. In these cases, the depth of the $973 \AA$ line core is similar to that of the O VI resonance line, and both form in the uppermost layers of the atmospheres. The Ne VII $973 \AA$ line is quite saturated so that it is not very strongly dependent on the neon abundance. This can be seen in

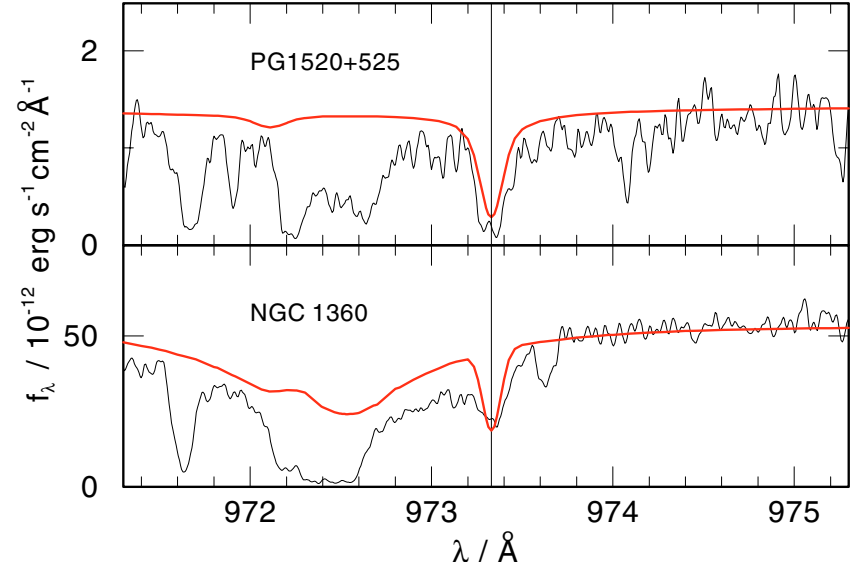

Fig. 6. Comparison of a PG 1159 star (PG 1520+525) and a "normal" hot hydrogen-rich central star (NGC 1360). The Ne VII $973.3 \AA$ line is also detectable in NGC 1360, which has a solar, i.e. much lower neon abundance.

Table 3. Ne VII lines identified in UV (vacuum wavelengths) and optical (air wavelength) spectra of PG 1159 stars. The last column gives the excitation energy of the lower level of the line transition. Level energies for the 3853-3912 A multiplet are from Kelly (1987), but line positions determined from our observations. As a consequence, the uncertainty in Kelly's energy for the lower level is $x \lesssim 1600 \mathrm{~cm}^{-1}$.

\begin{tabular}{rllr}
\hline \hline$\lambda / \AA$ & Transition & Reference & $E / \mathrm{cm}^{-1}$ \\
\hline 973.3 & $2 \mathrm{p}^{1} \mathrm{P}^{\mathrm{o}}-2 \mathrm{p}^{2}{ }^{1} \mathrm{D}$ & Lindeberg (1972) & 214952 \\
\hline 1982.0 & & & \\
1992.1 & $3 \mathrm{~s}^{3} \mathrm{~S}-3 \mathrm{p}^{3} \mathrm{P}^{\mathrm{o}}$ & Wiese et al. (1966) & 978320 \\
1997.3 & & & \\
\hline 3643.6 & $3 \mathrm{~s}^{1} \mathrm{~S}-3 \mathrm{p}^{1} \mathrm{P}^{\mathrm{o}}$ & JK69 & 998250 \\
\hline 3853.3 & & & $1028386+x$ \\
3866.8 & & & $1028519+x$ \\
3873.2 & & & $1028519+x$ \\
3894.0 & $3 \mathrm{p}^{3} \mathrm{P}^{\mathrm{o}}-3 \mathrm{~d}^{3} \mathrm{D}$ & This paper & $1028775+x$ \\
3905.1 & & & $1028775+x$ \\
3912.3 & & & $1028775+x$ \\
\hline
\end{tabular}

Fig. 3 for the case of PG $1525+525$. In the respective panel we included a model profile with a $\mathrm{Ne}$ abundance reduced to the solar value, i.e. by a factor of 20 down to $0.1 \%$. The equivalent width is reduced by only a factor of about two. In contrast, the optical $3634 \AA$ line reacts sensitively on abundance changes. In all of our program stars in which we see this optical line, it would be undetectable if the neon abundance were only solar.

On the other hand, the Ne VII $973 \AA$ line opens the possibility to detect neon in very hot stars even at a solar neon abundance level. In Fig. 6 we show the FUSE spectrum of the very hot $\left(T_{\text {eff }}=110 \mathrm{kK}, \log g=5.6\right.$; Hoare et al. 1996) hydrogenrich central star of NGC 1360 which has (at least roughly) a solar abundance composition. The Ne VII line is clearly visible and it is well matched by a model calculated with solar element abundances. 


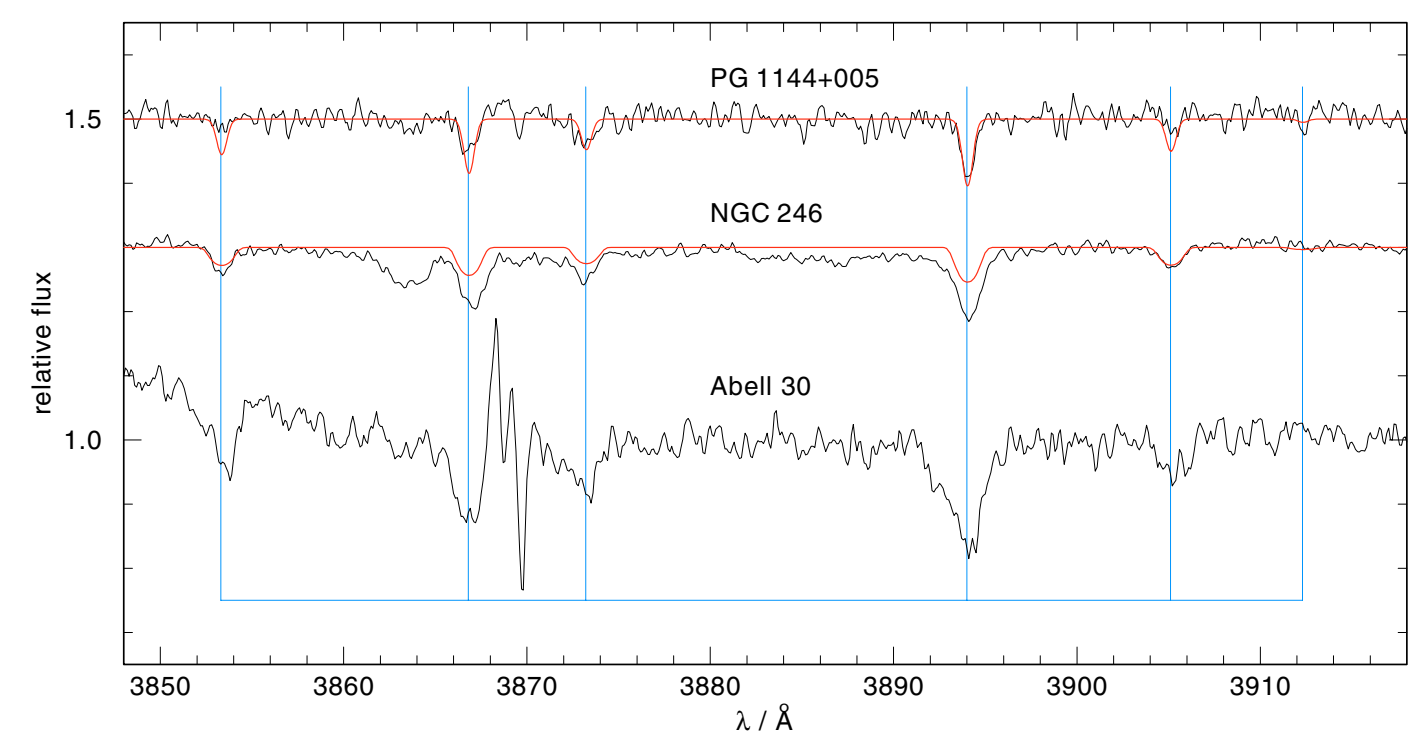

Fig. 7. Identification of the Ne VII $3 \mathrm{p}^{3} \mathrm{P}^{\mathrm{o}}-3 \mathrm{~d}^{3} \mathrm{D}$ multiplet in three PG 1159 stars. The vertical bars indicate the observed line positions which lie about $6 \AA$ r redward of the expected position when using Kelly's energy levels. Overplotted are the models for PG 1144+005 and NGC 246. The emission/absorption features between 3869-3870 $\AA$ in Abell 30 are residuals from the subtraction of a nebular emission from [Ne III] $3869.06 \AA$.

A new Ne VII multiplet, consisting of six lines in the 3853-3912 ̊̊ range, is identified in our VLT spectra of three PG 1159 stars (Fig. 7), which means the first detection of this multiplet in any stellar spectrum. It is the $3 \mathrm{p}^{3} \mathrm{P}^{\mathrm{o}}-3 \mathrm{~d}^{3} \mathrm{D}$ transition, whose exact line positions were unknown up to now because of uncertain level energies. We have taken the energy levels from Kelly (1987). If we shift the whole corresponding multiplet by $-6 \AA$ then all predicted line positions fall into observed absorption features within $\pm 1 \AA$. This enables us to determine the line positions to an accuracy of about $0.2 \AA$ (vertical bars in Fig. 7). This is a nice example how high-resolution stellar spectroscopy can improve our knowledge of atomic data.

As a side note, it is interesting to recall that Heap (1975) mentioned the presence of a line feature at $3867.5 \AA$ in her spectrum of NGC 246. Now, after three decades, it is evident that she saw for the first time the $3866.8 \AA$ line of that Ne VII multiplet.

\section{Comparison of observed and theoretical neon line profiles}

Let us discuss the strengths of the Ne VII lines in the FUSE and VLT spectra and compare them to our models. As already mentioned, all the synthetic line profiles were computed with a neon abundance of $2 \%$. We begin with the objects whose FUV spectra are displayed in Fig. 1 (and in more detail in Fig. 3), and then we turn to the objects for which we have only the optical VLT data (Figs. 4, 5 and 7).

As already emphasized, the $973 \AA$ line is potentially contaminated by interstellar $\mathrm{H}_{2}$. It is therefore mandatory to carefully examine its possible contribution to the photospheric neon line. We will see that the $973 \AA$ line in most objects appears significantly broader than predicted by our models. Possible origins are discussed in Sect. 4.2.

\subsection{Discussion of individual objects}

RX J2117.1+3412: the $973 \AA$ line is located in the broad and shallow emission part of the C III P Cygni profile. The heavilysaturated interstellar $\mathrm{H}_{2} \mathrm{P}(1)_{11-0}$ line coincides with the predicted Ne VII profile, rendering it difficult to confirm or refute its presence. There is substantial residual absorption, however, that is much broader than predicted by the photospheric model, and which cannot be explained by interstellar absorption.

K1-16: the weak narrow absorption seen at $973.4 \AA$ is consistent with absorption by IS $\mathrm{H}_{2}$. There is substantial residual absorption, however, that is even broader than that seen in RX J2117.1+3412. The $3644 \AA$ line was already detected by WR94.

PG 1144+005: the $973 \AA$ A line profile is affected by the red wing of the interstellar $\mathrm{HI}$ Ly $\gamma$ absorption line. The IS $\mathrm{H}_{2}$ absorption is quite weak and offset significantly in wavelength. The neon line is very strong and significantly broader than the computed profile. Increasing $\log g$ within reasonable limits does not improve the fit, see also the discussion of the next object. We also identify the $3644 \AA$ line. It is rather weak and well fit by the synthetic profile. The strongest components of the Ne VII 3853-3912 A multiplet are also identified and we obtain a good model fit.

PG 1520+525: the $973 \AA$ line is very strong and the synthetic profile fits very well. The observed line profile is in strong contrast to the previous object. Both stars have the same $T_{\text {eff. }}$ The surface gravity of PG $1144+005$ is lower by one dex but, quite unexpectedly, its $973 \AA$ line is much broader. The IS $\mathrm{H}_{2}$ contamination is a bit stronger than in PG $1144+005$, but is still a minor contributor to the overall profile.

PG 1159-035: here we also see a strong $973 \AA$ line, which is significantly broader than what we expect from the synthetic profile. The IS $\mathrm{H}_{2}$ contamination is not significant. This star's gravity is also lower than that of PG $1520+525$, but the line 
profile is much broader. The $3644 \AA$ line is not detectable in the VLT/1 spectrum of PG 1159-035 (Fig. 4), but easily identified, although being quite weak, in the higher-resolution VLT/2 spectrum (Fig. 5). Accordingly, the model profile is very weak, too. The Ne VII 3853-3912 A is detectable (not shown).

Longmore 4: as with RXJ2117.1+3412, the heavilysaturated IS $\mathrm{H}_{2}$ absorption coincides with and obscures the predicted Ne VII absorption. There is, however, very strong unexplained absorption that is blue-shifted from the predicted line center. Among all the VLT spectra, the $3644 \AA$ line of Longmore 4 is the strongest (together with NGC 246). However, the computed profile for Longmore 4 is much too weak. We believe that this is partly due to the fact that its temperature is significantly higher than previously determined. The observed strong O VI $3811 / 3834 \AA$ doublet is also in contrast to the weak computed emission and, hence, points into the same direction. Instead of $120 \mathrm{kK}, T_{\text {eff }}$ could be as high as that of NGC $246(150 \mathrm{kK})$. In the highest resolution spectrum (Fig. 5), the optical Ne VII line appears asymmetric, showing a blue wing that is broader than the red one. We think that this is due to wind effects. So the line equivalent width in this star is stronger than predicted also because of our static model assumption.

PG 1424-535 and PG 1707+427 are among the "cool" PG 1159 stars and our models predict rather weak $973 \AA$ A lines, because the ionization balance of neon shifts strongly in favor of lower ionization stages. $\mathrm{H}_{2}$ is weak but clearly detected in PG $1707+427$, and appears sufficient to explain the observed absorption at $973 \AA . \mathrm{H}_{2}$ is not convincingly detected in the spectrum of PG 1424-535; instead what is shown in Fig. 2 is a 3- $\sigma$ upper limit to the $\mathrm{H}_{2}$ column density. The resulting profile is consistent with the observed spectrum. The signal-to-noise of the FUSE spectra for both PG 1424-535 and PG 1707+427 is relatively low; better data are required to determine conclusively whether or not the predicted weak Ne VII absorption is present.

We have augmented Figs. 1-3 with the FUSE spectrum of H1504+65, which is the hottest PG 1159 star (200 kK). It shows strong Ne VII lines, both, in the FUV and the optical (W04).

We now turn to those objects for which only optical data are available (see Fig. 4). We remark in advance, that the O VI 3 s -3 p doublet at $3811 / 3834 \AA$ may be strongly enhanced in emission strength by a stellar wind, so that we cannot expect good fits of our models to these lines. This is most obvious in the case of Abell 30.

RX J0122.9-7521 is the second hottest PG 1159 star $\left(T_{\text {eff }}=180 \mathrm{kK}\right)$ after H1504+65 $(200 \mathrm{kK})$. The $3644 \AA$ line cannot be detected. Also, the predicted profile is very weak and shows that the line can escape detection despite the overabundance of Ne. When compared to H1504+65 this seems surprising. But the surface gravity of RX J0122.9-7521 is lower, favoring the shift of the neon ionization balance to ionization stages higher than Ne VII.

NGC 246 has the strongest $3644 \AA$ line, together with Longmore 4. Its equivalent width is significantly stronger than the computed one. Five out of the six components of the Ne VII 3853-3912 ̊ multiplet are identified and, again, the observed equivalent widths are larger. This suggests that the Ne abundance in both stars may be even higher than $2 \%$. We recall that a similar result was found for H1504+65 (see Sect. 1). Note that NGC 246 is rotating, perhaps with up to $v \sin i \approx 70 \mathrm{~km} \mathrm{~s}^{-1}$ (Rauch \& Werner 1997), which can partly explain the broad line profile.

Abell 21: the optical lines cannot be detected. The synthetic profiles show that, as in the case of PG 1159-035, the relatively high surface gravity shifts the ionization balance away from Ne VII to Ne VI.

Longmore 3: the observed $3644 \AA$ line is much stronger than the calculated one. In this case we think that either $T_{\text {eff }}(140 \mathrm{kK})$ is higher than assumed and/or that $\log g(6.3)$ is lower. Both would increase the calculated line strength. This is corroborated by the appearance of the O VI $3811 / 3834 \AA$ doublet: The calculated lines are in absorption whereas the observed ones are in emission. This also points to higher $T_{\text {eff }}$ and/or lower $\log g$.

PG 1151-029: the optical lines cannot be detected. The computed profiles show that they can escape detection in our VLT spectrum.

Abell 30 presents a strong $3644 \AA$ A line but nothing is seen in the model. As in the case of Longmore $4, T_{\text {eff }}$ is probably underestimated and/or $\log g$ is overestimated. Out of all objects presented here, the Ne VII 3853-3912 ̊ multiplet is most prominent in Abell 30. Again, the lines do not show up in the model, because its parameters are not appropriate.

NGC 7094 and Abell 43 are the two hybrid-PG 1159 stars in our sample. They are relatively cool so that only a weak $3644 \AA$ A line can be expected. Indeed, a very weak line is possibly present in NGC 7094, but we regard this as uncertain. It is not detectable in Abell 43.

MCT0130-1937 is the coolest object in our VLT sample $(90 \mathrm{kK})$. Consequently, the optical lines are not detectable.

\subsection{Summary of neon line analyses}

Table 4 summarizes the results. Ne VII lines were not detected in seven objects: RX J0122.9-7521, Abell 21, PG 1151-029, PG 1424-535, Abell 43, MCT0130-1937, and PG 1707+427. In all these cases one can neither exclude nor prove a neon overabundance, because the respective atomic levels are weakly populated due to photospheric parameters. In the other ten objects, neon lines are detected. A comparison with model profiles reveals a strong overabundance in some of these, however, a detailed abundance analysis still needs to be performed for all of them.

In six PG 1159 stars we have detected the Ne VII $973 \AA$ line. It is remarkable that the observed line profile in five of these stars is much broader than the synthetic profile (see Table 4). Can wind effects, neglected in our models, play a role? Probably not, at least not in all cases. While three of these five stars show P Cyg profiles, two don't: PG 1159-035 and PG 1144+005. Another explanation could be line broadening by pulsations. Four out of five of these broad-line PG 1159 stars are non-radial pulsators (identified in Table 4). PG 1159-035 is indeed the prototype of the GW Vir pulsators, and periodic UV 
Table 4. Summary of Ne VII line identifications in our program stars. "+" and "_" denote detection and non-detection, respectively. "broad" means that the detected line is much broader than the computed one. No entry means that no spectra are available. The second column denotes if the star is a pulsator $(+)$ or non-pulsator $(-)$. No entry means, that no photometric observations were performed or published.

\begin{tabular}{lcccc}
\hline \hline Object & Variable & $973 \AA$ & $3644 \AA$ & $3850-3910 \AA$ \\
\hline RX J0122.9-7521 & - & & - & - \\
RX J2117.1+3412 & + & broad & & \\
PG 1520+525 & - & + & & \\
PG 1144+005 & - & broad & + & + \\
NGC 246 & + & & + & + \\
PG 1159-035 & + & broad & + & + \\
Abell 21 & & & - & - \\
K1-16 & + & broad & + & \\
Longmore 3 & - & & + & - \\
PG 1151-029 & - & & - & - \\
Longmore 4 & + & broad & + & - \\
PG 1424+535 & - & - & & - \\
Abell 43 & & & - & - \\
NGC 7094 & & & + & - \\
Abell 30 & & & + & - \\
MCT0130-1937 & - & & - & - \\
PG 1707+427 & + & - & & \\
\hline
\end{tabular}

line profile shifts with an amplitude of the order $5 \mathrm{~km} \mathrm{~s}^{-1}$ were detected in HST/STIS spectra (Dreizler \& Werner 2004). The Ne VII line in the FUSE spectrum would require a much larger amplitude. This is not necessarily a contradiction, because the weak HST-UV lines (mostly C IV and O VI lines) form in deeper regions of the atmosphere than the strong Ne VII $973 \AA$ line, and different depths may experience different pulsation velocities. Hence, pulsation could explain the broad line profiles in PG 1159-035 and the other broad-line PG 1159 stars except for one object, namely PG 1144+005. Several observation campaigns have failed to detect light variability in this star (see, e.g., Steininger et al. 2003).

It is worth mentioning that the unusual width of the Ne VII $973 \AA$ line in PG 1159-035 and PG 1144+005 comes along with a similar effect in their O VI resonance doublets. These doublets have optical depths comparable to the neon line and also show broader and shallower cores than the models. In contrast, the only star which has a narrow Ne VII $973 \AA$ line (that is compatible with the model), also exhibits an $\mathrm{O}$ VI line that is fitted by the model. An interpretation of the oxygen doublet in the other four broad-line PG 1159 stars is problematic because it appears as a prominent P Cyg profile.

\section{Discussion and concluding remarks}

In order to discuss the implications of our results we briefly describe our current understanding about the origin of PG 1159 stars. We refer the reader to the work of Herwig (2001) for more details.

PG 1159 stars are hot post-AGB stars which have suffered a late (or very late) helium-shell flash during their first descent from the AGB. This event transforms a post-AGB star (or a white dwarf) back to an AGB star ("born-again" AGB star) and it then descends from the AGB for a second time. The He-shell flash is the origin of the H-deficiency that characterizes the PG 1159 stars (and their immediate progenitors, the [WC]-type central stars).

The surface abundance pattern of PG 1159 stars essentially reflects the composition of the matter that is located between the $\mathrm{H}$ - and He-burning shells (intershell region) of thermally pulsing AGB stars. Intershell matter is helium-dominated (ash of $\mathrm{CNO}$ hydrogen-burning), but also strongly enriched by $\mathrm{C}$ and $\mathrm{O}$ from the $3 \alpha$-process in the He-burning shell. The strong $\mathrm{C}$ and $\mathrm{O}$ intershell enrichment is the consequence of effective convective overshoot that dredges up $\mathrm{C}$ and $\mathrm{O}$ into the convective intershell. Evolutionary models predict a neon abundance of about $2 \%$ by mass in the intershell. This ${ }^{22} \mathrm{Ne}$ is produced in the He-burning shell from ${ }^{14} \mathrm{~N}$ (which itself was produced previously by CNO-burning) via

${ }^{14} \mathrm{~N}(\alpha, \gamma){ }^{18} \mathrm{~F}\left(\mathrm{e}^{+} v\right){ }^{18} \mathrm{O}(\alpha, \gamma){ }^{22} \mathrm{Ne}$.

Our result, that the neon abundance in some objects is strongly overabundant, is therefore in qualitative agreement with theoretical predictions.

This view is further supported by recent observations which reveal s-process signatures in the surface composition of PG 1159 stars. While it is impossible to detect the enrichment of s-process elements in these hot objects (because of the lack of atomic data for high ionization stages), we have instead found a deficiency of iron in all examined PG 1159 stars (Miksa et al. 2002; Werner et al. 2003a). Obviously, Fe was transformed to heavier elements in the intershell region of the AGB star by $n$-captures from the neutron source ${ }^{13} \mathrm{C}(\alpha, n){ }^{16} \mathrm{O}$.

How is the $\mathrm{He} / \mathrm{C} / \mathrm{O} / \mathrm{Ne}$-rich intershell matter mixed to the surface of the star, and what happens to the H-rich envelope matter? This is interesting in order to discuss the observed nitrogen abundance in PG 1159 stars.

As a consequence of the He-shell flash a convection zone develops above the He-burning shell that eventually reaches the $\mathrm{H}$-rich envelope. Hydrogen is ingested and burned. According to Iben \& MacDonald (1995) large amounts of nitrogen can be produced $(5 \%)$ by the reaction chain

${ }^{12} \mathrm{C}(\mathrm{p}, \gamma){ }^{13} \mathrm{~N}\left(\mathrm{e}^{+} v\right){ }^{13} \mathrm{C}(\mathrm{p}, \gamma){ }^{14} \mathrm{~N}$

because ample ${ }^{12} \mathrm{C}$ is available in the intershell from $3 \alpha$ burning. However, calculations by Herwig (2001), with a more extensive nuclear network, show that no fresh nitrogen is produced. Instead of ${ }^{13} \mathrm{C}$ reacting with protons to form ${ }^{14} \mathrm{~N}$, it reacts with ${ }^{4} \mathrm{He}$ to form ${ }^{16} \mathrm{O}$, so that protons are burned in the chain

${ }^{12} \mathrm{C}(\mathrm{p}, \gamma){ }^{13} \mathrm{~N}\left(\mathrm{e}^{+} v\right){ }^{13} \mathrm{C}(\alpha, \mathrm{n}){ }^{16} \mathrm{O}$.

Here we have an effective neutron source which activates further n-capture nucleosynthesis (Herwig et al. 2003).

If no fresh $\mathrm{N}$ is produced, and if all $\mathrm{N}$ from previous CNO cycling was transformed into ${ }^{22} \mathrm{Ne}$, then the question remains unanswered, why a considerable fraction of PG 1159 stars (six objects) exhibits nitrogen on the $1 \%$ level. As 
a coincidence, these six N-rich stars are within our sample (PG 1144+005, PG 1159-035, Abell 30, PG 1707+427, Abell 43, NGC 7094). The first four of these positively show Ne VII lines, i.e., the neon must have been produced from CNO-cycle generated nitrogen.

The hybrid-PG 1159 stars are a special case. We know four such objects which still have considerable amounts of residual surface hydrogen (two are in our sample: Abell 43 and NGC 7094). According to Herwig (2001) this is the result of a He-shell flash experienced by an AGB-star immediately before its departure from the AGB. They also show residual nitrogen, which seems natural in this event. The hybrid-PG 1159 stars essentially display an intershell matter abundance pattern like the other PG 1159 stars, however, it is diluted by hydrogen-rich envelope matter. So we also would expect a neon-enrichment, but this is difficult to conclude from our spectra.

In summary, the strong neon enrichment found in some PG 1159 stars is qualitatively in agreement with evolutionary models for stars which suffered a late He-shell flash. The surface chemistry of PG 1159 stars is that of intershell matter in the precursor AGB stars. The neon enrichment and the irondeficiency caused by s-processing strongly support this scenario.

Acknowledgements. UV data analysis in Tübingen is supported by the DLR under grant 50 OR 0201. R.N. acknowledges support by a PPARC advanced fellowship. J.W.K. is supported by the FUSE project, funded by NASA contract NAS5-32985. We thank Falk Herwig (LANL) for helpful discussions on evolutionary aspects, and Dr. Kramida (NIST) and Prof. Kunze (University of Bochum) for their advice on the Ne VII $973 \AA$ line identification. This research has made use of the SIMBAD Astronomical Database, operated at CDS, Strasbourg, France. The UVES spectra used in this analysis were obtained as ESO Service Mode runs. The interstellar absorption analysis was done using the profile fitting procedure Owens.f developed by M. Lemoine and the FUSE French Team.

\section{References}

Abgrall, H., Roueff, E., Launay, F., Roncin, J. Y., \& Subtil, J. L. 1993a, A\&AS, 101, 273

Abgrall, H., Roueff, E., Launay, F., Roncin, J. Y., \& Subtil, J. L. 1993b, A\&AS, 101, 323

Ballester, P., Boitquin, O., Modigliani, A., et al. 2003, UVES Pipeline User's Manual, ESO, Garching

Dekker, H., D’Odorico, S., Kaufer, A., Delabre, B., \& Kotzlowski, H. 2000, Proc. SPIE, 4008, 534

Dreizler, S., \& Heber, U. 1998, A\&A, 334, 618

Dreizler, S., \& Werner, K. 2004, A\&A, submitted

Dreizler, S., Werner, K., \& Heber, U. 1997, in Planetary Nebulae, ed. H. J. Habing, \& H. J. G. L. M. Lamers (Kluwer), IAU Symp., 180, 103
Heap, S. R. 1975, ApJ, 196, 195

Hébrard, G., Lemoine, M., Vidal-Madjar, A., et al. 2002, ApJS, 140, 103

Herwig, F. 2001, Ap\&SS, in Low Mass Wolf-Rayet Stars: Origin and Evolution, ed. R. Waters, A. Zijlstra, \& T. Blöcker, 275, 15

Herwig, F., Lugaro, M., \& Werner, K. 2003, in Planetary Nebulae, ed. S. Kwok, M. Dopita, \& R. Sutherland (ASP), IAU Symp., 209, 85

Hoare, M. G., Drake, J. J., Werner, K., \& Dreizler, S. 1996, MNRAS, 283,830

Iben, I. Jr., \& MacDonald, J. 1995, in White Dwarfs, ed. D. Koester, \& K. Werner (Berlin: Springer), Lecture Notes in Physics, 443, 48

Johnston, W. D., \& Kunze, H.-J. 1971, Phys. Rev. A, 4, 962 (JK69)

Kelly, R. L. 1987, Journal of Physical and Chemical Reference Data, 16, Suppl. No. 1

Kruk, J. W., \& Werner, K. 1998, ApJ, 502, 858

Kruk, J. W., Howk, J. C., \& André, M. 2002, ApJS, 140, 19

Lang, J. 1983, J. Phys. B, 16, 3907

Lindeberg, S. 1972, Uppsala Univ. Inst. Phys., Report UUIP-759, 1

Miksa, S., Deetjen, J. L., Dreizler, S., et al. 2002, A\&A, 389, 953

Moos, H. W., Cash, W. C., Cowie, L. L., et al. 2000, ApJL, 538, 1

Morton, D. C. 2003, ApJS, 149, 205

Napiwotzki, R., Christlieb, N., Drechsel, D., et al. 2001, AN, 322, 411

Napiwotzki, R., Christlieb, N., Drechsel, D., et al. 2003, ESO Messenger, 112, 25

Rauch, T., \& Werner, K. 1997, in The Third Conference on Faint Blue Stars, ed. A. G. D. Philip, J. Liebert, \& R. A. Saffer (Schenectady, NY: L. Davis Press), 217

Sahnow, D. J., Moos, H. W., \& Ake, T. B. 2000, ApJL, 538, 7

Steininger, B., Paparo, M., Virághalmy, G., Zsuffa, D., \& Breger, M. 2003, in Interplay of Periodic, Cyclic and Stochastic Variability in Selected Areas of the H-R Diagram, ed. C. Sterken, ASP Conf. Ser., 292, 237

Werner, K. 2001, Ap\&SS, in Low Mass Wolf-Rayet Stars: Origin and Evolution, ed. T. Blöcker, L. B. F. M. Waters, \& A. A. Zijlstra, 275, 27

Werner, K., \& Heber, U. 1991, A\&A, 247, 476

Werner, K., \& Rauch, T. 1994, A\&A, 284, L5 (WR94)

Werner, K., \& Wolff, B. 1999, A\&A, 347, L13

Werner, K., Heber, U., \& Hunger, K. 1991, A\&A, 244, 437

Werner, K., Hamann, W.-R., Heber, U., et al. 1992, A\&A, 259, L69

Werner, K., Wolff, B., Pakull, M., et al. 1996a, in Supersoft Xray Sources, ed. J. Greiner (Berlin: Springer), Lecture Notes in Physics, 472, 131

Werner, K., Dreizler, S., \& Heber, U., et al. 1996b, A\&A, 307, 860

Werner, K., Dreizler, S., Koesterke, L., \& Kruk, J. W. 2003a, in Planetary Nebulae, ed. S. Kwok, M. Dopita, \& R. Sutherland (ASP), IAU Symp., 209, 239

Werner, K., Deetjen, J. L., Dreizler, S., et al. 2003b, in Stellar Atmosphere Modeling, ed. I. Hubeny, D. Mihalas, \& K. Werner, ASP Conf. Ser., 288, 31

Werner, K., Rauch, T., Barstow, M. A., \& Kruk, J. W. 2004, A\&A, 421, 1169 (W04)

Wiese, W. L., Smith, M. W., \& Glennon, B. M. 1966, Atomic transition probabilities, Vol. 1, NSRDS-NBS4, US Dept. of Commerce Young, P. R., Del Zanna, G., Landi, E., et al. 2003, ApJS, 144, 135 